\title{
A martingale-based temporal analysis of pre-earthquake anomalies at Jiuzhaigou, China, in the period of 2009-2018
}

\author{
Ling Lin ${ }^{1, *}$, Xiangzeng Kong ${ }^{1}$, and Nan $\mathrm{Li}^{2}$ \\ ${ }^{1}$ Fujian Normal University, 350022 Fuzhou Fujian, China \\ ${ }^{2}$ Fujian Agriculture and Forest University, 350002 Fuzhou Fujian, China
}

\begin{abstract}
Many studies on the relationship between outgoing longwave radiation (OLR) data observed by National Oceanic and Atmospheric Administration Satellites (NOAA) with strong earthquakes are based on a certain zone or a couple of earthquakes. But it's hard to know if the algorithm works by looking at just one earthquake. In this paper, after algorithm analysis of OLR signals based on martingale theory during the 10 years from 2009 to 2018, a time series analysis of re-anomaly screening for the 10 years' data is proposed. The experimental results show that this method can be more effective in the statistical analysis of historical data and further improve the reliability of prediction.
\end{abstract}

\section{Introduction}

The most of the cause of earthquakes is the rupture of a faults zone [1], there are many earthquakes occurred just because of the area on the fault-melt, and the thermal omen is observed at the same time [2]. The fault zone showing rapid shear heating of fault gouge might preserve stress magnitude accommodated the thermal pressurization during the earthquake [3]. Scholars have proposed that 2017 Jiuzhaigou earthquake is delayed by the Wenchuan earthquake and the former event possibly results from the south-eastward movement of the Bayankala block, which in turn arises from the collision of Indian-Australian Plate and the Eurasian Plate [4]. The Jiuzhaigou earthquake occurred in the region among the eastern segment of the East Kunlun, Minjiang, and Huya faults.

Studies show that there are thermal anomalies and the change in electrical state [2], [5] and co-seismic slip and fault [6], [7] when an earthquake occurs. The study of the infrared data of seismic satellites in different periods around the world shows that many large areas of infrared radiation data are abnormally warming before strong earthquakes [8]-[11].

Many artificial intelligence analysis methods have found anomalies in the thermal infrared data before the earthquake, which include data mining, machine learning, and pattern recognition. The log-likelihood method can be used to detect changes in multi-dimensional data sets, Kuncheva [12] gave a log-likelihood justification for Kullback-Leibler (K-L) distance and Hotelling's Tsquare test for equal means, which use a single round of k-means clustering instead of density approximation, Song et al. [13] defined a statistical test call density test to deciding if the observed data points are sampled from the underlying distribution. Ho and Wechsler [14], [15] proposed a martingale framework for detecting changes in exchangeable data streams, which showed that it can be effective in unlabelled data streams. Severo and Gama propose to compose a regression predictor, a Kalman filter and Cumulative Sum of Recursive Residual (CUSUM) to detected changes [16]. The predictor can continuously detect the error of the regression model, whose significant increase is taken for an abnormal change. A Bayesian approach for earthquake forecasting model was put forward by Marzocchi et al. [17].

Other methods involving analysis of thermal properties and statistical analysis of standard deviation have been proposed to find anomalies in the OLR data before earthquake [6], [18]. Chakraborty et al. studied the effects of seismic events on OLR in the M7.3 Nepal earthquake that occurred on May 12, 2015, and a previous large earthquake in on Apri25, 2015[19]. They used the method of Eddy field calculation mean to find the anomaly and the relation with the tectonic plate boundary. Xiong et al. utilized a data mining technique based on wavelet transform to analyse the anomalies of longwave emission radiation (OLR) data; they propose that there is a certain correlation between the abnormal information found and earthquakes [20]. The OLR, from several decades, provides a significant pattern of transient anomalies preceding earthquakes [6], [7], [19], [21]-[23]. The OLR anomaly corresponds to a large area of ground coverage and coincides with the main epicentral zone [6], and primary was used to research Earth radiative budget and climate. An increase in radiation and a transient change in OLR were proposed to be related to thermodynamic processes in the atmosphere over seismically active regions [21]. The fault may create huge thermal energy which captured by NOAA and converted to OLR data.

*Corresponding author: linling@ @jnu.edu.cn 
Table 1. The list of seismic events occurred in grid 5th at Jiuzhaigou between April and August of 2008 - 2019.

\begin{tabular}{|c|c|c|c|c|c|c|}
\hline No & Date & latitude & longitude & depth & mag & place \\
\hline 1 & $2018 / 6 / 29$ & 32.2028 & 104.6042 & 10 & 4.8 & 23km SSE of Long'an, China \\
\hline 2 & $2017 / 8 / 19$ & 33.0454 & 103.8128 & 10 & 4.4 & 46km WSW of Yongle, China \\
\hline 3 & $2017 / 8 / 10$ & 33.4554 & 103.7869 & 10 & 4.1 & 46km WNW of Yongle, China \\
\hline 4 & $2017 / 8 / 9$ & 33.2638 & 103.7895 & 10 & 4.8 & $41 \mathrm{~km} \mathrm{~W}$ of Yongle, China \\
\hline 5 & $2017 / 8 / 8$ & 33.1926 & 103.8552 & 9 & 6.5 & 36km WSW of Yongle, China \\
\hline 6 & $2017 / 4 / 10$ & 32.4156 & 104.7126 & 30.36 & 4.5 & 17km E of Long'an, China \\
\hline 7 & $2013 / 7 / 26$ & 33.002 & 104.92 & 18.9 & 4.6 & 6km S of Linjiang, China \\
\hline 8 & $2012 / 4 / 29$ & 32.637 & 104.988 & 35 & 4.3 & Sichuan-Gansu border region, China \\
\hline 9 & $2010 / 8 / 18$ & 32.146 & 104.717 & 10 & 4.2 & Sichuan-Gansu border region, China \\
\hline 10 & $2010 / 6 / 30$ & 32.313 & 104.908 & 32.9 & 4.8 & Sichuan-Gansu border region, China \\
\hline 11 & $2009 / 5 / 14$ & 32.291 & 104.914 & 35.5 & 4.5 & Sichuan-Gansu border region, China \\
\hline 12 & $2009 / 4 / 30$ & 32.214 & 104.923 & 24.5 & 4.3 & Sichuan-Gansu border region, China \\
\hline
\end{tabular}

Based on the previous studies, in this paper, we take the OLR data of Jiuzhaigou from 2008 to 2019 as an example to study the abnormal results obtained by martingale theory algorithm, and conduct further analysis of time series to discover the relationship between time-series data changes and earthquake occurrence. We proposed a method which called the Martingale and SEA Algorithm (MSA) algorithm base on our previous work, which is reference Martingale theory [15], [24] and the Geometric Moving Average (GMA) method. The algorithm obtains the abnormal from OLR raw dataset.

The earthquake preparation process may start 1-30 days ago, Event. These multidisciplinary data provide a synopsis of the atmosphere/ionospheric variations related to tectonic activity. People found before an earthquake, there are some abnormal occurs, but they are instability and hard to find.

After the abnormal signal is extracted, it needs to be further analysed. Generally, the occurrence of an earthquake has a preparation process may start 1-30 days ago [19], [25]. To validate their result and to find out those characteristics of OLR in other aspects, we apply some statistical studies based on the data of Jiuzhaigou, China, 2009-2018 in this study, and we were more focus on the Ms6.5 earthquake at Aug 8th, 2017 to conduct the time series analysis.

\section{Data Source and Pre-processor}

\subsection{Data Source}

Outgoing Longwave Radiation (OLR) measured at the top of the atmosphere [21], which are not directly measured from the raw data using a separate algorithm [26]. In this paper, the OLR dataset used was from National Oceanic and Atmospheric Administration satellites (ftp://ftp.cpc.necp.noaa.gov/precip/noaa18_olr), which provides two data sets each day, one containing the OLR data for the "afternoon" satellite (i.e. equator crossing time 1430 0230 LST, currently "NOAA-14") and one day set for the "morning" satellite (i.e. equator time 0630 1830 currently "NOAA-15"). The data are stored in $144 \times 72$ arrays in ASCII, and each value represents the OLR flux on a 2.5 degrees latitude/longitude (units are $\mathrm{W} / \mathrm{m}^{2}$ ). As shown in Fig. 1, according to the data format, we divide the Jiuzhaigou fault zone into 9 grid areas, where each area indicated $2.5^{\circ}$ latitude $\times 2.5^{\circ}$ longitudes. The five-point start in the middle expressed the epicentre of the earthquake on August8, 2017 in Jiuzhaigou.

We have studied OLR signals in Jiuzhaigou region from 2009 to 2018 and conducted time series analysis based on martingale theory algorithm in our previous research. The information about these earthquakes is from the website of U.S. Geological Survey, which is part of the National Earthquake Hazards Reduction Program (NEHRP) led by the National Institute of Standards and Technology (NIST).

(https://earthquake.usgs.gov/earthquakes/)

The epicentre of the Jiuzhaigou earthquake was $33.193^{\circ} \mathrm{N} 103.855^{\circ} \mathrm{E}$, corresponding to the grid 5 th, the longitude was between $102.605^{\circ} \mathrm{E}$ and $105.105^{\circ} \mathrm{E}$, and the dimensions were $34.443^{\circ} \mathrm{N}$ and $31.943^{\circ} \mathrm{N}$. No. 5 in table 1 lists the information about this earthquake.

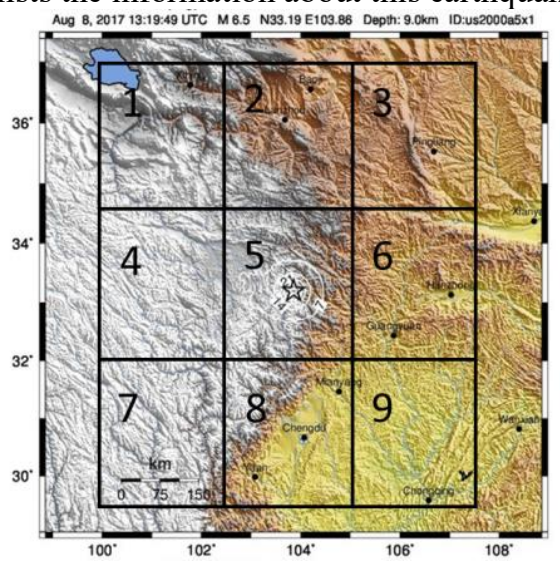

Fig. 1. The $2.5 * 2.5$ Grid Diagram of the Jiuzhaigou Earthquake as the Epicentre on August 8, 2017. The grid in this paper based on time series research focused on the 5th grid in the middle. 
Table 1 lists 12 seismic events that occurred in grid 5 (Fig. 1) between April and August from 2008 to 2019. This list of seismic events is filtered by the rule that if there are several earthquakes event on the same day, left the only one which is the highest magnitude and the shallowest depth. For example, on August 8 and 9, 2018, there were 5 earthquakes and 6 earthquakes respectively, and we chose one of each according to the guidelines. We chose those earthquakes which have occurred in the period from April to August annually. After processing by the martingale theory algorithm, the year-end data is vulnerable to other data during the year, and some nodes at the beginning of the year are used as the starting point for the algorithm.

\subsection{Data Pre-processing}

Before we start the experiment, we need to process the data first. NOAA data is stored in a file-per-day manner, which is relatively complete and does not require cleaning, but sometimes it is missing. First, the following strategies are adopted for data normalization: 1) Three days of data loss were found in the data of 10 years, they are 20080514, 20080515 and 20090509. We copied the data from the previous day to supplement the complete data. 2) About the in OLR set, the missing values are identified as "-9999". This situation is rare, usually one or a couple day, so we replaced it with the previous day's data. 3) Concerning leap years, we uniformly normalized the deletion on February 29. After the processing of the above steps, the 10 -years OLR database studied is a relatively complete and regular time series with 365 data points per year. Secondly, OLR data are generally superposition of several signals: weather effect, diurnal temperature difference, artificial noises, or some underground activities. To minimize the impact of data noise, we utilized the night time dataset. The third is to grid the earthquake region. Taking the epicentre of Ms 6.5 earthquake that occurred in Jiuzhaigou area on August 8, 2017, that is $36 \mathrm{~km}$ WSW of Yongle as the No. 5 in label 1, divided into 9 grids, which is convenient for spatial and temporal analysis. In this paper, sequence analysis is carried out with grid 5 as the core to discuss the relationship between pre-seismic anomalies of the OLR data and earthquake events.

\section{Methodologies}

\subsection{A martingale method}

The Martingale theory has been widely used in investment optimization, decision-making optimizations, and survival analysis. In [14], [24], Ho and Wechsler proposed a Martingale framework for detecting changes and found the feasibility of the martingale method for detecting changes in unlabelled data streams. In our previous work, we applied the martingale theory algorithm proposed by Ho et al. to analysed the OLR dataset, and it was effective to obtain the abnormal information from OLR data [5], [7], [27]. In probability theory, a martingale is a sequence of random variables (i.e., a stochastic process) for which, at a particular time, the conditional expectation of the next value in the sequence, given all prior values, is equal to the present value.

Basic on the martingale arithmetic, we combined the GMA/EWMA control charts to improve the results for precision. The benefit of GMA/EWMA Martingale method is that it can obtain better precision and recall than the pure Martingale method [15].

Consider an unlabelled time series example the OLR dataset, set $Z=\left\{z_{1}, z_{2}, \ldots, z_{i-1}\right\}$, and $z_{i}$ represent the current point. The strangeness number defined as through the clustering algorithm to statistics the distance between $t_{i}$ and $T$, so the distance of the set closest to the point can be obtained, expressed as $s_{i}$, $s_{i}=\left\|z_{i}-m\right\|$ where $\|*\|$ means distance metric result and $m_{k}$ is expressed as the $k$ th cluster sets. $\hat{p}_{i, k}$ is computed from the function as follow:

$$
\hat{p}_{i, k}\left(\mathrm{Z} \cup\left\{z_{n}\right\}, \theta_{n}\right)=\frac{\#\left\{j \mid s_{j}>s_{i}\right\}+\theta_{i, k} \#\left\{j \mid s_{j}=s_{i}\right\}}{i}
$$

Where parameter $\theta_{i, k} \in[0,1]$ is a random value, and $i=1,2, \ldots, n, j=1,2, \ldots, i$. A family of Martingales, indexed by $\varepsilon \in[0,1]$ and referred to as the randomized power Martingale [15], is defined as follow:

$$
M_{n}^{(\varepsilon)}=\prod_{i=1}^{n} \varepsilon \hat{p}_{i}^{\varepsilon-1}
$$

Where $\hat{p}_{i}$ is computed using equation (1). The serial $M_{n}^{(\varepsilon)}$ is the result of the Martingale algorithm. Base on this, we propose a new method to measure the degree of change in data streams, and set $\varepsilon=0.64$, call CD-Value as follows:

$$
C D_{n}^{(\varepsilon)}=\frac{\sum_{k=1}^{100} \prod_{i=1}^{n} \varepsilon \hat{p}_{i, k}^{\varepsilon-1}}{100}
$$

If the computed serial data $C D_{n}$ value is very high and exceeds some value, this could correspond to an abnormal condition. Therefore, the threshold $h$ needed to be settled to stop the process. The condition $C D_{n} \geq h$ can be used to determine whether an abnormal change has been detected. Here we set $h=1000$, and if a change is found, re-initializing the calculation of the equation.

\subsection{Time analysis and anomaly detection}

From the previous step, the OLR data transform to a serial of CD-Value dataset, which mines valuable abnormal change information from the original OLR data. The calculating process of CD-value dataset is a Moving Window Martingale Theory (MWMA) actually, so we call it as MWMA value or CD value (Change Detected) sets. However, it is hard to get really useful 
information simply by analysing the earthquake of a single curve of these CD-Value sets. We tried to find the rule by studying the $\mathrm{CD}$ data of 10 years and using statistical methods. This paper takes grid 5 in figure 1 of the OLR data set in Jiuzhaigou region from 2009 to 2018 as the research object.

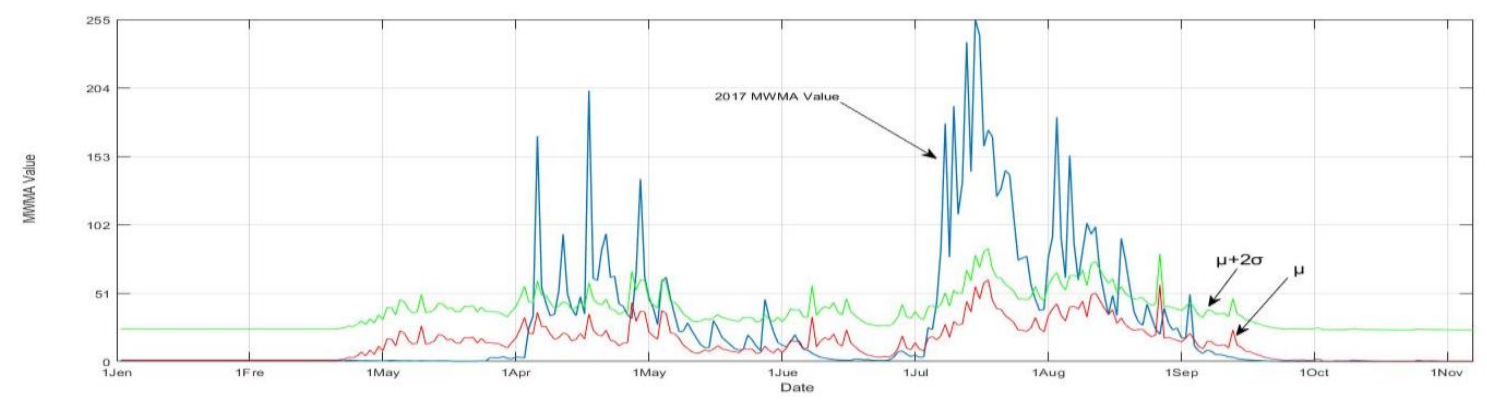

Fig. 2 Compare 2017 MWMA Value in Jiuzhaigou with the mean of 10 years $\mu$ and the interquartile range $\mu+2 \sigma$.

First of all, the important thing is to identify and define anomalies. Consider the ten years matrix $T=\left\{t_{i j} \mid i=2009, \ldots, 2018 ; j=1,2, \ldots, 365\right\}$, calculate the mean of it as follows:

$$
\mu_{j}=\frac{\sum_{i=2009}^{2018} t_{i j}}{10}
$$

The next step is to calculate the standard deviation $\sigma$ of this matrix. To find out a proper criterion for the anomaly definition, we check the empirical probability distribution of the 10-year MWMA value. In Figure 2, shows the contrast between the blue, red and green three curves, demonstrate the MWMA value of 2017 years in Jiuzhaigou, and mean value $\mu$ and $\mu+2 \sigma$ of 10 years, respectively. The conventional method to identify anomaly data from a certain data set is using the $\mu+2 \sigma$ threshold. However, in Figure 2, it could be found that the energy distributions are quasi-normal or skew, and the extremely large MWMA value can seriously bias the mean and the $\sigma$. Therefore, here we utilize median and interquartile range, which are more robust estimations, to define the anomaly. As a result, there are 905 anomalies if the threshold is $\mu$ and 314 anomalies if it is $\mu+2 \sigma$.

\section{Experimental Results}

A disturbance that cannot be recognized by the naked eye can be obtained by data mining of OLR data source. Figure 3 shows the comparison before and after data processing. In the picture above, the blue wave line is the OLR raw data; it is hard to get the regularity and abnormality. Meanwhile, the picture below is the after martingale algorithm processing, it is completely different from the original data curve. The MWMA value captured the abnormal information. The red dashed lines in Figure 3 indicated twice earthquake event in
2017, from left to right correspond to No.6 and No.5 in table 1. As the Figure 3 the MWMA value image shows, the MWMA value very small before April 10, after the April 10 earthquake, the value of MWMA fluctuated slightly, and then all the way down, until the value began to rise in July, and gradually reached the peak, and then fell to the next data climb, just happened on August 8 The magnitude 6.4 earthquake. This shows that the exception of the OLR signal was successfully acquired.
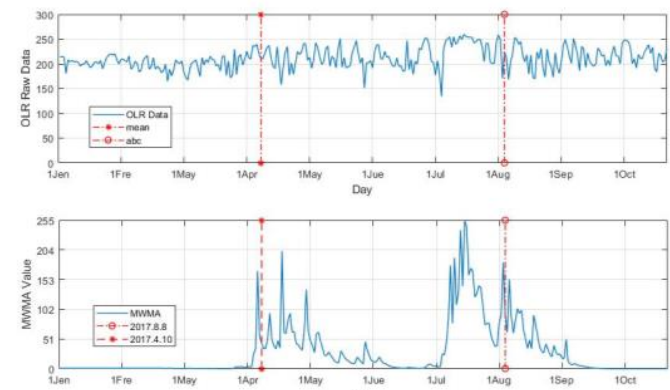

Fig.3 The MWMA value mines and amplifies abnormal variation values of OLR source data in Jiuzhaigou, 2017.

About the martingale method, if we set different the value of $\varepsilon$ would get a different result. For example, in the research of the earthquake events in Lushan and Wenchuan in 2008 set $\varepsilon=0.82$ is better choice relatively. Figure 4 shows the different value of $\varepsilon$ getting various. Because of the parameter $\varepsilon$ influenced the degree and amplitude of those abnormal points. Before we study a seismic region, we need to judge and choose the parameters of the area. Figure 4 shows 3 value of parameter $\varepsilon$, they are some typical case. The red line represent the day of $\mathrm{Ms}=6.5$ earthquake. If $\varepsilon=0.54$, the amount of abnormal values detected is smaller. As the parameter value increases, the magnitude of the abnormal detection begins to increase, but when it gets too large, some small earthquake trigger abnormal may be ignored. 

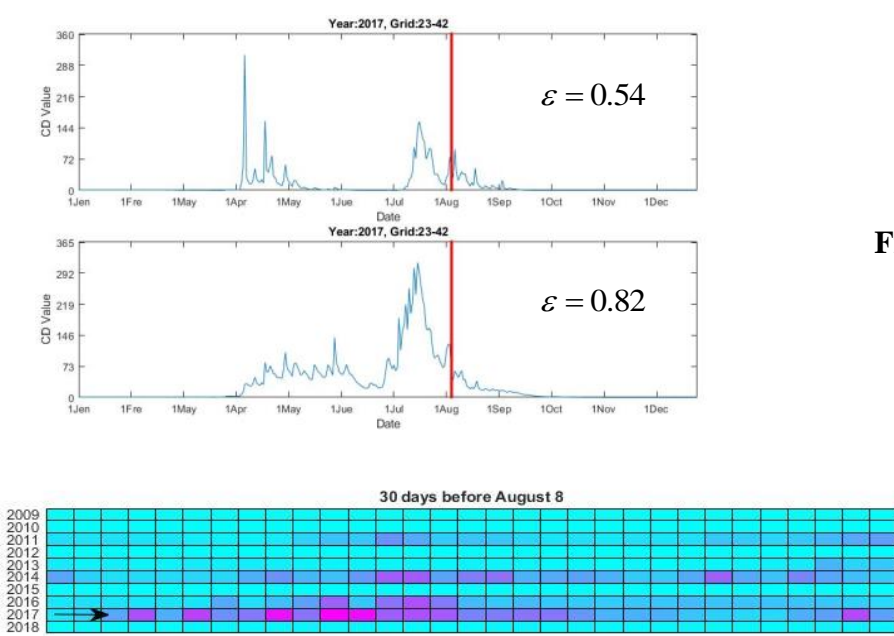

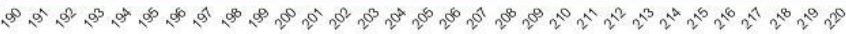

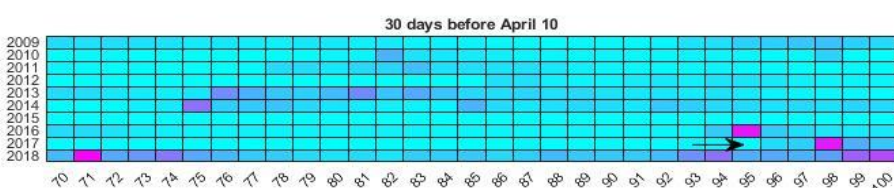

Fig. 5 Before earthquake 30-day thermodynamic diagram of Twice earthquake in 2017

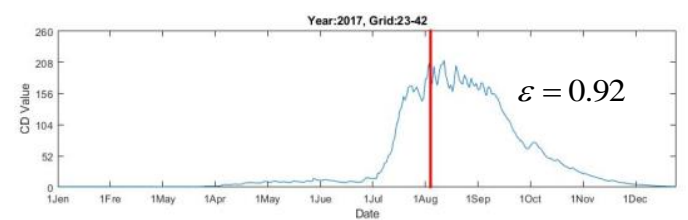

Fig.4 The effect of setting different $\varepsilon$ on the results of CDValue
In figure 4 as the $\varepsilon=0.82$, the abnormal values detected before and after the April 10 earthquake is significantly reduced, until $\varepsilon=0.92$ the abnormal value becomes very small.

There are much other reasons may trigger the WMWA abnormal. We couldn't make an earthquake early warning as soon as the abnormal appear. Even in a non-earthquake year, there may appear a similarity abnormal curve. So, we have studied the relations of multi-years. Furthermore, to investigate the occurrence and of the MWMA anomalies of ten years, and apply the above method of identification abnormal, and compare them to other normal years. Figure 5 shows the thermodynamic diagram of the twice earthquake in Jiuzhaigou in 2017. In Figure 5, the colour indicates the magnitude of the MWMA value, the purple means the value bigger, and the cyan means smaller. The vertical is 2008-2019 years and the horizon is 30 days before the earthquake on Aug. 8, 2017, and the most right square indicates the earthquake day is the 220th day, so there are 30 squares in the coordinates per row. In Figure 5, the black arrow is referring to 2017.

The similar situation occurs on April 10, 2017. Compare to August 8, the earthquake is smaller, the purple also occurs, and have almost a pure purple square, that is the value is very big. There are 5 purple squares in Figure 5(b), one belongs to 2017, 3 belongs to 2018 years. Of course, we found in 2018 occurs the Ms=4.8 earthquake. Compared the same period with other years, purple squares are the most visible, and the quantity of the purple square is the most. And that the $\mathrm{Ms}=4.8$ earthquake happened in June 2018, which was a small sign of things to come. In Figure 5, the graph on the right is the CD-Value curve corresponding to the year of the earthquake on the left, that is the year indicated by the arrow, and the red line shows the date of the earthquake, which can be compared with each other to increase the credibility of some earthquake signs.

\section{CONCLUSIONS}

During the period from April to June in 2009 to 2018, a total of 12 earthquakes of magnitude 4 or above occurred in the Jiuzhaigou area, and the Ms 6.5 earthquake occurred on August 8, 2017. Firstly, we screen the parameters of this region, and then extract the abnormal MWMA value of martingale theory algorithm from the OLR data of 10 years, and then carry out time-series analysis on this basis. The results show that the multiyear lateral comparison can help to confirm the reliability of earthquakes. We still have a lot of work to do. For example, if we first make difference another algorithm for stationary time series data of source data, we can eliminate the noise caused by seasonal temperature changes. The algorithm works well inland, so how do you tell if an earthquake is happening on an island or off the coast when the temperature is out of control. We will further study other signals such as GPS.

\section{References}

1. Y. Gui-Xi et al., Focal mechanism solutions and seismogenic structure of the 8 August 2017 M7.0 Jiuzhaigou earthquake and its aftershocks, northern Sichuan, CHINESE J. Geophys. Ed., no. 10, pp. 4083-4097, (2017).

2. D.-F. Liu, K.-Y. Peng, W.-H. Liu, L.-Y. Li, and J.-S. Hou, Thermal omens before earthquakes, ACTA Seismol. Sin., no. 6, pp. 710-715, 12(1999).

3. L.-W. Kuo, V. Luzin, P. C. Chen, E. C. Yeh, and K. F. Ma, Deformation textures in the principal slip zone of the Chelungpu fault, Taiwan, and its implication for stress change during the seismic cycle, in AGU Fall Meeting Abstracts, (2018). 
4. K. Jia et al., Did the $2008 \mathrm{Mw} 7.9$ Wenchuan earthquake trigger the occurrence of the $2017 \mathrm{Mw}$ 6.5 Jiuzhaigou earthquake in Sichuan, China?, J. Geophys. Res. Solid Earth, no. 4, pp. 2965-2983, 123(2018).

5. Jiang X, Kong X, Guo G. A method of identifying and analyzing Outgoing Long-wave Radiation anomalies before earthquake[C], MATEC Web of Conferences. EDP Sciences, 189: 11001, (2018).

6. D. Ouzounov, D. Liu, K. Chunli, G. Cervone, M. Kafatos, and P. Taylor, Outgoing long wave radiation variability from IR satellite data prior to major earthquakes, Tectonophysics, no. 1-4, pp. 211-220, 431(2007).

7. X. Kong, N. Li, L. Lin, P. Xiong, and J. Qi, Relationship of Stress Changes and Anomalies in OLR Data of the Wenchuan and Lushan Earthquakes, IEEE J. Sel. Top. Appl. Earth Obs. Remote Sens., no. 8, pp. 2966-2976, 11(2018).

8. S. Pulinets and D. Ouzounov, LithosphereAtmosphere-Ionosphere Coupling (LAIC) model An unified concept for earthquake precursors validation, J. Asian Earth Sci., no. 4-5, pp. 371382, 41(2011).

9. S. A. Pulinets, D. Ouzounov, A. V. Karelin, K. A. Boyarchuk, and L. A. Pokhmelnykh, The physical nature of thermal anomalies observed before strong earthquakes, Phys. Chem. Earth, no. 4-9, pp. 143153, 31(2006).

10. A. Verma and S. K. Saraf, 4-Thiazolidinone-A biologically active scaffold, Eur. J. Med. Chem., no. 5, pp. 897-905, 43(2008).

11. V. Tramutoli, V. Cuomo, C. Filizzola, N. Pergola, and C. Pietrapertosa, Assessing the potential of thermal infrared satellite surveys for monitoring seismically active areas: The case of Kocaeli (Izmit) earthquake, August 17, 1999, Remote Sens. Environ., no. 3-4, pp. 409-426, 96(2005).

12. L. I. Kuncheva, Change detection in streaming multivariate data using likelihood detectors, IEEE Trans. Knowl. Data Eng., no. 5, pp. 1175-1180, 25(2011).

13. X. Song, M. Wu, C. Jermaine, and S. Ranka, Statistical change detection for multi-dimensional data, in Proceedings of the 13th ACM SIGKDD international conference on Knowledge discovery and data mining, pp. 667-676, (2007).

14. S.-S. Ho and H. Wechsler, Detecting Changes in Unlabelled Data Streams Using Martingale. in IJCAI, pp. 1912-1917, (2007).

15. M. Intelligence and $\mathrm{H}$. Wechsler, A Martingale Framework for Detecting Changes in Data Streams by Testing Exchangeability, IEEE Trans. PATTERN Anal. Mach. INTELL, no. 12, pp. 2113-2127, 32(2010)

16. M. Severo and J. Gama, Change detection with kalman filter and cecum, in International
Conference on Discovery Science, pp. 243-254, (2006).

17. W. Marzocchi, J. D. Zechar, and T. H. Jordan, Bayesian forecast evaluation and ensemble earthquake forecasting, Bull. Seismol. Soc. Am., no. 6, pp. 2574-2584, 102(2012).

18. L. Wu, K. Qin, and S. Liu, GEOSS-based thermal parameters analysis for earthquake anomaly recognition, Proc. IEEE, no. 10, pp. 2891-2907, 100(2012).

19. S. Chakraborty, S. Sasmal, S. K. Chakrabarti, and A. Bhattacharya, Observational signatures of unusual outgoing longwave radiation (OLR) and atmospheric gravity waves (AGW) as precursory effects of May 2015 Nepal earthquakes, J. Geodyn., pp. 43-51, 113(2018).

20. P. Xiong et al., Study of outgoing longwave radiation anomalies associated with Haiti earthquake, Nat. Hazards Earth Syst. Sci., no. 10, pp. 2169-2178, 10(2010).

21. D. Ouzounov et al., Atmosphere-Ionosphere Response to the M9 Tohoku Earthquake Revealed by Joined Satellite and Ground Observations. Preliminary results, Ref arxiv. org/abs/1105.2841, DOI, 10(2011).

22. S. Chakraborty, S. K. Chakrabarti, S. Sasmal, and A. Bhattacharya, Monitoring of unusual Outgoing Longwave Radiation (OLR) during the May, 2015 Nepal earthquake using satellite observations, in 42nd COSPAR Scientific Assembly, 42(2018).

23. G. U. O. Xiao, Z. Yuan-Sheng, Z. Mei-Jiao, S. Wen-Rong, and W. E. I. Cong-Xin, Variation characteristics of OLR for the Wenchuan earthquake, Chinese J. Geophysics., no. 6, pp. 980988, 53(2010).

24. Ho, Shen-Shyang. A martingale framework for concept change detection in time-varying data streams. Proceedings of the 22nd international conference on Machine learning. ACM, (2005).

25. P. Han et al., Statistical analysis of ULF seismomagnetic phenomena at Kakioka, Japan, during 2001-2010, J. Geophys. Res. Sp. Phys., no. 6, pp. 4998-5011, 119(2014).

26. G. Ohring and A. Gruber, Satellite radiation observations and climate theory, in Advances in Geophysics, Elsevier, pp. 237-304, 25(1983).

27. X. Kong, Y. Bi, and D. H. Glass, "Detecting seismic anomalies in outgoing long-wave radiation data," IEEE J. Sel. Top. Appl. Earth Obs. Remote Sens., no. 2, pp. 649-660, 8(2015). 